\title{
Diffusion process and formation of super-spin-glass state in soft magnetic Fe/Pt system
}

\author{
D. Aurongzeb ${ }^{\text {a) }}$ and M. Holtz \\ Department of Physics, Texas Tech University, Lubbock, Texas 79409-1051 \\ and The NanoTech Center, Texas Tech University, Lubbock, Texas 79409-1051 \\ Latika Menon \\ Department of Physics, Northeastern University, Boston, Massachusetts 02115
}

(Received 22 May 2006; accepted 5 July 2006; published online 28 August 2006)

\begin{abstract}
We report results on surface and micromagnetic structures of Fe thin films consisting of a $\mathrm{Pt}$ underlayer. We use atomic force microscopy to study the surface structure evolution of the Fe films as a function of annealing time at an annealing temperature of $800{ }^{\circ} \mathrm{C}$. Power spectral density analysis shows saturation in roughness exponent after $15 \mathrm{~min}$ of annealing. However, lateral correlation length and roughness continue to increase for up to $45 \mathrm{~min}$. At high annealing temperature, the authors find two separate phase correlation lengths and a single surface correlation length indicating super-spin-glass state in the system. (C) 2006 American Institute of Physics.
\end{abstract}

[DOI: $10.1063 / 1.2335977]$

Ferromagnetic nanostructures are currently the subject of intense research activity due to their future technological applications. Numerous experimental and theoretical studies have been performed for various two- and three-dimensional arrangements of magnetic nanostructures having different degrees of structural and magnetic disorder. ${ }^{1-3}$ For example, grains with in the nanoscale thin films can act as a dense nanomagnet and their random orientation can dictate their magnetic behavior. Since grains with the thin films are randomly oriented they can become soft ferromagnetic despite the presence of some hard ferromagnetic phases.

Recently FePt films have attracted a great deal of attention because of their application in high-density magnetic recording. ${ }^{4,5}$ The order-disorder transition temperature of FePt films is typically around $600{ }^{\circ} \mathrm{C}$. Therefore, a postannealing procedure is required for their fabrication. Most studies on FePt thin films focus on structural characterization in multilayer films with the goal towards obtaining the hard magnetic phase of FePt alloy. Though there are few studies available on nanoparticle formation due to annealing, ${ }^{6}$ we find no report detailing surface structure evolution and its correlation with magnetic domain formation, as Fe/Pt system is structurally stable, and the focus is always the high coercivity phase.

In this letter, we report surface structure and magnetic domain evolution in $\mathrm{Fe}(10 \mathrm{~nm})$ thin films containing a $\operatorname{Pt}(5 \mathrm{~nm})$ under layer. We find that the thickness is ideal for studying surface-interface interaction without making $\mathrm{Fe} / \mathrm{FePt}$ islands. Evolution of surface structure is studied by means of atomic force microscopy imaging (AFM) as a function of annealing time. We report power spectral density (PSD) analysis of the AFM images and compare the results with the magnetic microstructures studied using magnetic force microscopy (MFM) and "bulk" magnetization measurements.

A $5 \mathrm{~nm}$ thin film of Pt was deposited on $\mathrm{Si}(111)$ wafer followed by a $10 \mathrm{~nm}$ layer of Fe in a thermal evaporator. The

\footnotetext{
a) Author to whom correspondence should be addressed; electronic mail: deeder.aurongzeb@ttu.edu
}

base pressure was $\sim 10^{-6}$ Torr and the deposition rate was $\sim 2 \AA / s$. Thickness of the Fe and Pt layers was controlled by a quartz thickness monitor located near the substrate holder. Sample temperatures remained below $100{ }^{\circ} \mathrm{C}$ during the deposition. The films were annealed at $800{ }^{\circ} \mathrm{C}$ inside a convectional furnace for 15, 30, and $45 \mathrm{~min}$. After annealing, the sample topography was characterized using an atomic force microscope (DI 3100). X-ray diffraction was used to examine structural phase. The corresponding micromagnetic structure was imaged using a magnetic force microscope (DI 3100). The results were compared with bulk magnetization as a function of applied magnetic field, measured using an alternating gradient force magnetometer.

Figure 1(a) shows two-dimensional (2D) AFM scan of the as-deposited $10 \mathrm{~nm}$ thin Fe film on the $\mathrm{Si}(111)$ wafer with $5 \mathrm{~nm}$ Pt under layer. In the image, one clearly observes the formation of clusters. The typical size of the cluster is 300 nm. Figures 1(a)-1(d) show the increase in cluster size as a function of annealing time as revealed in the 2D AFM scans. The increase in cluster size suggests a substantial diffusion process in the Fe film at the annealing temperature of $800{ }^{\circ} \mathrm{C}$. It may be added that further increase in annealing time does not lead to any noticeable change in cluster size,

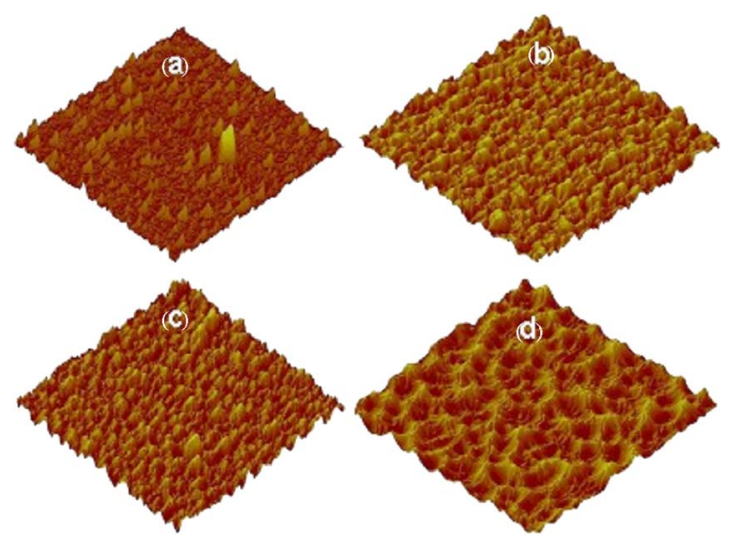

FIG. 1. (Color online) AFM image $\left(5 \times 5 \mu \mathrm{m}^{2}\right)$ of surface evolution at different annealing times: (a) as-deposited film, (b) $15 \mathrm{~min}$, (c) $30 \mathrm{~min}$, and (d) $45 \mathrm{~min}$. 

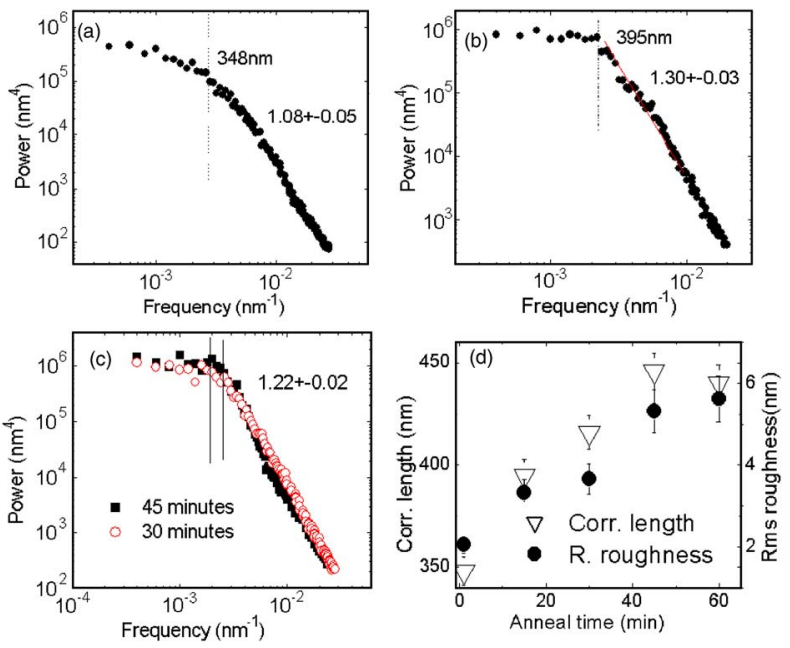

FIG. 2. (Color online) PSD plots and summary for various annealing times: (a) as-deposited, (b) $15 \mathrm{~min}$ annealing, (c) $30 \mathrm{~min}$ annealing, and $45 \mathrm{~min}$ annealing. (d) Dependence of correlation length and rms roughness as a function of annealing time.

indicating saturation. We believe that this is due to nanocluster formation at the surface and the interfaces. As the clusters get bigger due to surface and interface diffusion cluster can no longer grow as the grain boundaries meet.

We have analyzed the above images using a power spectral density analysis. A brief description of the PSD process is given here (details can be found in Ref. 7). PSD analysis allows us to identify scaling behavior according to

$$
\operatorname{PSD}(k) \propto k^{-\gamma},
$$

where the exponent $\gamma$ is related to the roughness exponent, $\alpha=(\gamma-d) / 2$, where $d=1$ in our analysis. ${ }^{8}$ Based on the above PSD procedure, we have obtained the PSD for all four AFM images, as demonstrated in Figs. 2(a)-2(c). Several images were taken at different magnifications for consistency. The roughness exponent calculations were also checked with height-height correlation function; however, PSD method is shown to be the most reliable. ${ }^{9}$

We interpret results using the theory of kinetic roughening. ${ }^{10}$ Several approaches have been developed to relate the growth dynamics with roughness exponent. One of the models is based on the Wolf-Villian linear diffusion model, ${ }^{11}$

$$
\frac{\partial h}{\partial t}=R_{D}-\nu \nabla^{4} h+\eta,
$$

where function $h(x, y)$ is a measured surface variable, such as topographical height in $\mathrm{AFM},(x, y)$ is the position at which the measurement is made, $R_{D}$ is the random deposition rate or, in our case, coalescence rate at surface and the interface, $\nu$ is a constant, and $\eta$ is a roughening term with stochastic characteristics. The $\nu \nabla^{4} h$ term describes smoothening by surface diffusion. In this model, the value for the roughness scaling exponent $\alpha=1$. One attempt to make surface diffusion term a conserved quantity is to add a nonlinear term to Eq. (2). The corresponding term is given by $\lambda \nabla^{2}(\nabla h)^{2}$ where $\lambda$ is a constant, ${ }^{12}$ generally associated with the growth velocity. The nonlinear term takes into account steps acting as a source or sink of atoms on a growing surface. For our case, an additional source of roughness is $\mathrm{Fe}-\mathrm{Pt}$ interface itself where interdiffusion takes place between the
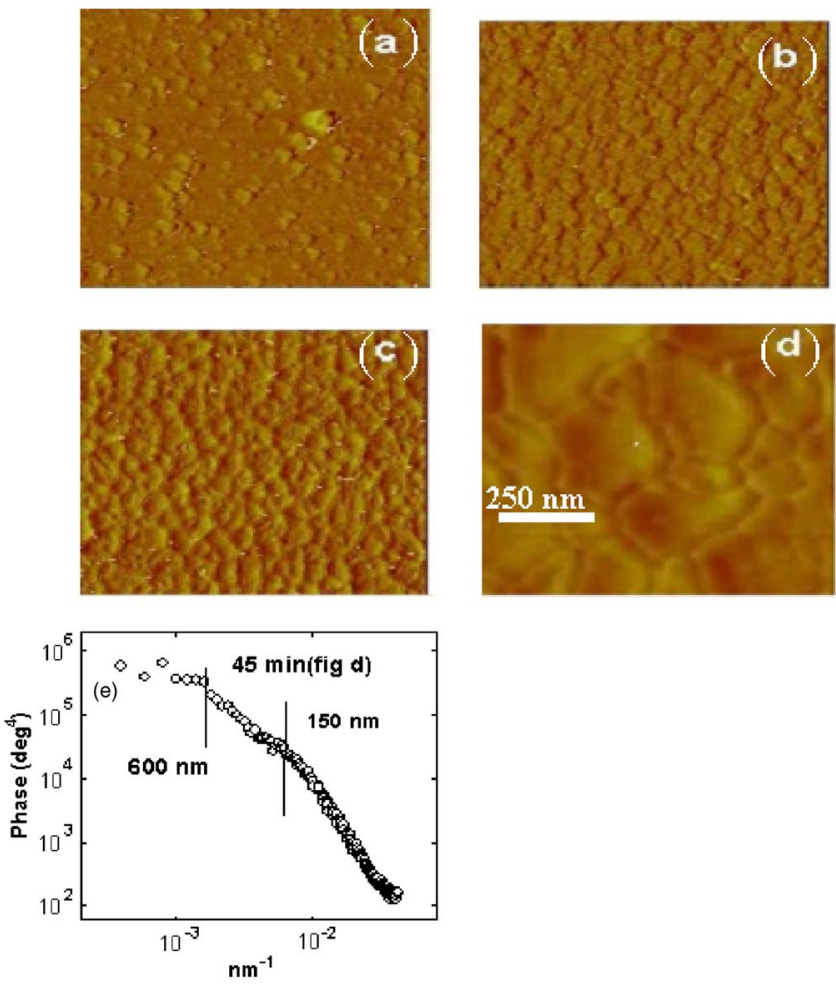

FIG. 3. (Color online) MFM image as a function of annealing time: (a) as-deposited, (b) $15 \mathrm{~min}$, and (c) $45 \mathrm{~min}$. (d) Smaller scan area (1 $\left.\times 1 \mu \mathrm{m}^{2}\right)$ of 45 min annealed sample. The domain walls are clearly visible. (e) Phase power spectrum showing domain correlation lengths. Observe two correlation lengths. Observe phase correlation length is larger than the surface correlation length in Fig. 2(d).

two materials. The theoretical exponent value including the nonlinear term is $3 / 2$.

The linear slope in PSD spectra of Fig. 2 provides the scaling exponent in Eq. (1) and subsequently $\alpha$. For the as deposited case, it may be noted that the roughness exponent is $\sim 1$. Therefore the surface process can be described by linear diffusive model of Eq. (2). The deposition temperature is substantially lower than the typical FePt alloying temperature, ${ }^{13}$ therefore, we do not expect interdiffusion to influence the surface properties. At this stage we expect surface processes to be mediated by Fe diffusion and coalescence during deposition. As we anneal the samples at $800{ }^{\circ} \mathrm{C}$, we expect surface diffusion and $\mathrm{Fe}-\mathrm{Pt}$ interdiffusion to influence the surface properties. These nonlinear effects are reflected in the higher roughness exponent, $\sim 1.2$ in the PSD analysis. This may be explained as arising due to development of magnetic order in the samples, possibly due to formation of magnetic FePt alloy phase in the sample. Formation of this phase is indicated in x-ray diffraction results(not shown) of our samples, which show FePt [111] lines.

In the PSD spectra of Fig. 2, cutoff to zero slope corresponds to $k_{c}=1 / \rho_{c}$ where $\rho_{c}$ is the correlation length between the surface clusters. In Fig. 2(d), we plot the correlation length as a function of annealing time. It may be noted here that increased cluster size in turn leads to increased surface roughness. For comparison, rms roughness values obtained from AFM images are also plotted in Fig. 2(d) showing the increasing trend.

The MFM image for the as-deposited sample [Fig. 3(a)] does not indicate domain formation. This suggests very weak magnetization. This result may be compared with magnetization versus field measurements. For the as-deposited to AlP license or copyright, see http://apl.aip.org/apl/copyright.jsp 


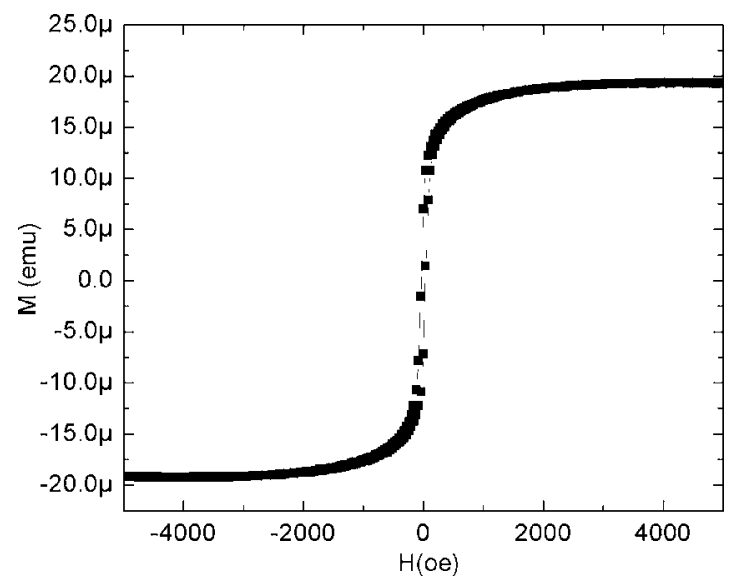

FIG. 4. Magnetization vs field curve for the Fe/Pt sample after annealing for $15 \mathrm{~min}$.

sample the coercivity is very small, $\sim 2 \mathrm{Oe}$, typical of $\mathrm{Fe}$ thin films. ${ }^{14}$ It may be mentioned that the coercivity is small in both parallel and perpendicular orientations of the magnetic field. After annealing for $15 \mathrm{~min}$, the MFM image shows formation of domains [see Fig. 3(b)]. It may be noted that this MFM image is clearly distinct from the corresponding topographic AFM image in Fig. 1(b). This confirms that the image in Fig. 3(b) is indeed due to the magnetic signal from magnetic exchange interaction. The typical domain size is of the order of $300-350 \mathrm{~nm}$. With increased annealing time, both domain size and domain wall thickness are found to be independent of annealing time. Figure 3(c) shows the MFM image of the sample annealed for 45 min while Fig. 3(d) shows the smaller scan area of the same. The image shows that shape is stabilized toward hexagonal shape. However, the shapes are of various sizes. This is interesting because AFM data image and correlation function show that the surface domains are uniform and the cutoff length is around $450 \mathrm{~nm}$. While magnetic correlation function in Fig. 3(e) shows two phase correlation lengths. According to the random anisotropy model ${ }^{15}$ when the random field is greater than the exchange field, the magnetic vector in this case points along the intraparticle anisotropy axis. As the interparticle exchange increases the system assumes correlated super-spin-glass state. ${ }^{16,17}$ The state is clearly seen in our system as we annealed the samples for longer time. We have two types of field based on the following equation: ${ }^{16}$

$$
\text { Corr }_{\text {phase }}=D \cdot H / H_{\text {random }} \text {. }
$$

The phase correlation lengths from Fig. 4(e) are 150 and $600 \mathrm{~nm}$. The surface particle diameter is $\sim 470 \mathrm{~nm}$ which is very close to the correlation length. Therefore, the ratios between exchange correlation and random correlation are 0.33 and 1.34. The intraparticle exchange increases with annealing time as the surface cluster density increases. As seen in Fig. 1 the clusters are almost touching each other by $15 \mathrm{~min}$. Detail MFM image at higher annealing time [Fig. 3(d)] shows that these clusters actually form magnetic domain walls of size of $\sim 50 \mathrm{~nm}$. This is an indication that magnetic domains are not due to surface structure only but due to complex exchange interaction between surface and the interface. As previously pointed out by Loffler et al. ${ }^{18}$ that as particle diameter becomes bigger, actual interaction model should include domain wall effect. The effect of interface is not really surprising due to the fact that certain amount of interdiffusion will take place at this temperature and time frame. In fact, certain hard magnetic phase is also reported at this temperature range. ${ }^{19}$

Domain formation in the microstructure is also consistent with bulk magnetization versus field measurements, which clearly shows a much larger coercivity in annealed samples compared to as-deposited films. For the $15 \mathrm{~min}$ annealed sample, the value of coercivity is $\sim 34$ Oe when the applied field is parallel to the surface of the film (Fig. 4). As in the case of the micromagnetic structure, bulk magnetization is also found to be independent of annealing time.

In conclusion, we have reported the surface and magnetic evolutions of thin $\mathrm{Fe}$ films deposited on a Pt layer. From a power spectrum analysis of atomic force microscopy images, we determine the value of roughness exponent to be $\sim 1$ for the as-deposited sample, consistent with a linear diffusive model. Upon annealing, the roughness exponent increases to about 1.2 indicative of nonlinear effects such as interdiffusion between $\mathrm{Fe}$ and the underlying $\mathrm{Pt}$ layer and coalescence effects leading to increased cluster size. Magnetic microstructure results obtained from MFM phase power spectrum show complex exchange mechanism between surface and interface.

The authors acknowledge support for this work from the National Science Foundation (ECS-0348156 and ECS0304224).

${ }^{1}$ S. Sun, C. B. Murray, D. Weller, L. Folks, and A. Moser, Science 287, 1989 (2000).

${ }^{2}$ J. Zhang, C. Boyd, and W. Lou, Phys. Rev. Lett. 77, 390 (1996).

${ }^{3}$ E. Lomba, F. Lando, and J. J. Weis, Phys. Rev. E 61, 3838 (2000).

${ }^{4}$ Z. Dai, S. Sun, and Z. Wang, Surf. Sci. 505, 325 (2002).

${ }^{5}$ S. C. Chou, C. C. Yu, Y. Liou, and Y. D. Yao, Phys. Status Solidi A 201, 1755 (2004)

${ }^{6}$ Masahiro Kitada, Thin Solid Films 146, 21 (1987).

${ }^{7}$ K. Zhu, V. Kuryatkov, B. Borisov, J. Yun, G. Kipshidge, S. Nikisin, H. Temkin, D. Aurongzeb, and M. Holtz, J. Appl. Phys. 95, 4635 (2004).

${ }^{8}$ J. Krug, Adv. Phys. 46, 139 (1997).

${ }^{9}$ J. Schmittbuhl, J.-P. Vilotte, and S. Roux, Phys. Rev. E 51, 131 (1995).

${ }^{10}$ A. L. Barabisi and H. E. Stanly, Fractal Concepts in Surface Growth (Cambridge University, Cambridge, 1995).

${ }^{11}$ D. Wolf and J. Villian, Europhys. Lett. 13, 389 (1990).

${ }^{12}$ Z. W. Lai and S. D. Sarma, Phys. Rev. Lett. 66, 2348 (1991).

${ }^{13}$ T. Thompson, B. Terris, M. Toney, S. Raoux, J. Baglin, S. Lee, and S. Sun, J. Appl. Phys. 95, 6738 (2004).

${ }^{14}$ M. Schneider, St. Müller-Pfeiffer, and W. Zinn, J. Appl. Phys. 79, 8578 (1996).

${ }^{15}$ E. M. Chudnovsky, J. Appl. Phys. 64, 5770 (1998).

${ }^{16}$ C. Binns, M. J. Maher, Q. A. Pankhurst, D. Kechrakos, and K. N. Trohidau, Phys. Rev. B 66, 184413 (2002).

${ }^{17}$ S. A. Koch, G. Palasantzas, T. Vystavel, J. Th. M. De Hosson, C. Binns, and S. Louch, Phys. Rev. B 71, 085410 (2005).

${ }^{18}$ J. F. Löffler, H.-B. Braun, and W. Wagner, Phys. Rev. Lett. 85, 1990 (2000).

${ }^{19}$ T. Shima, K. Takanashi, Y. K. Takahashi, K. Hono, G. Q. Li, and S. Ishio, J. Appl. Phys. 99, 033516 (2006). 